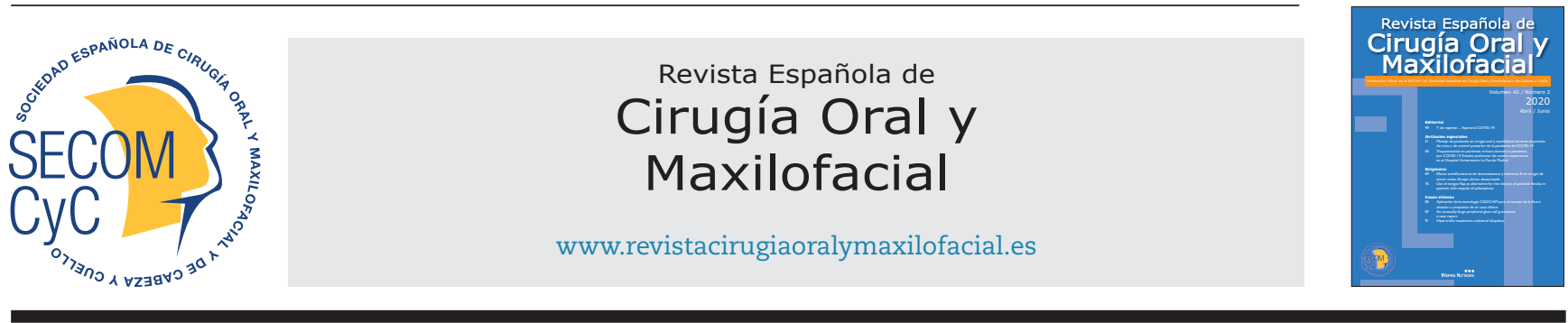

Artículo especial

\title{
Traqueotomías en pacientes críticos durante la pandemia por COVID-19. Estudio preliminar de nuestra experiencia en el Hospital Universitario La Paz de Madrid
}

\author{
José Luis del Castillo Pardo de Vera ${ }^{a}$, José Luis Cebrián Carreteroa, Jorge Guiñales Díaz \\ de Cevallos ${ }^{a}$, Pedro Garrido Ortega ${ }^{b}$, Jorge Noguera Tomás ${ }^{a}$, Jesús Manuel Muñoz Caro ${ }^{a}$, \\ Teresa González Otero ${ }^{a}$, María José Morán Soto ${ }^{a}$, Albert Malet Contreras ${ }^{a}$, Celia Sánchez \\ Gallego-Albertos ${ }^{a}$, Juan Carlos Figueira Iglesiasc, María José Asensio Martín', José Manuel \\ Añón Elizalde ${ }^{c}$ y Miguel Burgueño García ${ }^{a}$
}

aServicio de Cirugía Oral y Maxilofacial. Hospital Universitario La Paz, Madrid, España. 'bervicio Anestesiología y Reanimación. Hospital Universitario La Paz, Madrid, España. 'Servicio de Medicina Intensiva. Hospital Universitario La Paz, Madrid, España

INFORMACIÓN DEL ARTÍCULO

Historia del artículo:

Recibido: 30 de abril de 2020

Aceptado: 12 de mayo de 2020

Palabras clave:

COVID-19, traqueotomía, equipo de protección, riesgo de infección.
R E S U M E N

\begin{abstract}
El brote epidémico causado por el virus SARS-CoV-2 se encuentra plenamente activo en España. Alrededor del 10-15\% de los pacientes ingresados precisan cuidados en unidades de críticos, siendo intubados de forma prolongada y precisando la realización de traqueotomías. Se realiza un estudio observacional de las traqueotomías realizadas por nuestro Servicio de Cirugía Oral y Maxilofacial a pacientes COVID-19 de unidades de cuidados intensivos realizadas entre el 17 de marzo y el 17 de abril de 2020. El estudio analiza aspectos epidemiológicos y clínicos de los pacientes, el tipo de técnica quirúrgica empleada, el tiempo quirúrgico, el tipo de cánula empleada, las complicaciones postquirúrgicas y el seguimiento clínico de los pacientes.

Un total de 22 pacientes fueron sometidos a traquetomía reglada abierta. Fueron dieciocho hombres y cuatro mujeres de edades entre 40 y 77 años (64,9 años de media). En todos los casos la realización de traqueotomía fue como consecuencia del proceso pulmonar por la neumonia bilateral COVID-19. Dos pacientes presentaron un neumotórax en el postoperatorio inmediato como complicación, un paciente falleció durante la realización del procedimiento y otro tras su llegada a la Unidad de Cuidados Intensivos tras la realización de la traqueotomía. A pesar de que la traqueotomía es una técnica quirúrgica reglada, las características especiales de los pacientes COVID-19 hacen de este procedimiento una situación crítica por la inestabilidad pulmonar y la rápida desaturación del paciente. Todo ello obliga a la realización del procedimiento por facultativos con experiencia para disminuir el tiempo quirúrgico y poder enfrentarse a cualquier eventualidad.
\end{abstract}

*Autor para correspondencia:

Correo electrónico: jldelcastillopardo@gmail.com (José Luis del Castillo Pardo de Vera).

DOI: 10.20986/recom.2020.1150/2020

1130-0558/C 2020 SECOM. Publicado por Inspira Network. Este es un artículo Open Access bajo la licencia CC BY-NC-ND (http:// creativecommons.org/licenses/by-nc-nd/4.0/). 
Tracheotomies in critical patients during the COVID-19 pandemia. Preliminary study of our experiences at La Paz University Hospital of Madrid

\section{A B S T R A C T}

Keywords:

COVID-19, tracheostomy, protective equipment, risk of infection.
The outbreak caused by the SARS-CoV-2 virus is currently very active in Spain. Many infected people still require to be hospitalized. Around 10-15\% of hospitalized patients require intensive care, where they are intubated for a prolonged period, needing tracheotomies some weeks after the intubation.

We will be conducting an observational study of the tracheotomies performed by our oral and maxillofacial Department to COVID-19 patients on intensive care units between March $17^{\text {th }}$ and April $17^{\text {th }}, 2020$. This study will be analyzing the patients' epidemiological and clinical aspects, surgical technique employed, surgical time, type of cannula used, postoperative complications and the patients' clinical monitoring.

A total of 22 patients underwent open elective tracheotomy. There were twenty-two males and three females aged between 40 and 77 (mean: 64,9 years-old). In all cases tracheotomy was carried out due to pulmonary process caused by COVID-19 bilateral pneumonia. Two patients presented pneumothorax in the immediate postoperatory care as a complication, one perished during the procedure and another did so after arriving to the Intensive Care Unit after the tracheotomy surgery.

Even though tracheotomy is a ruled surgical technique, the special characteristics of COVID-19 patients make of this procedure a critical situation, mainly due to lung instability and quick desaturation of the patients. This requires the surgery to be carried out by experienced physicians in order to reduce operative time and to be able to react to any eventualities that may arise.

\section{INTRODUCCIÓN}

El primer caso de coronavirus en Madrid se registró el pasado 25 de febrero, afectando a un joven de 24 años que había estado de viaje por el norte de Italia, primer foco de COVID-19 en Europa. Desde entonces, el número de contagios del COVID-19 ha aumentado progresivamente. En el Hospital Universitario La Paz de Madrid se registraron los primeros casos de COVID-19 en la Comunidad de Madrid; en un principio se ingresaron en el Hospital Carlos III, pero cuando se requirieron cuidados críticos y el número de ingresos superó la capacidad de este hospital, fue necesario activar nuevas plantas en el Hospital Universitario La Paz. En la actualidad (abril de 2020), los pacientes enfermos por COVID-19 ocupan la mayor parte de las camas del hospital y la capacidad de las UVI se ha multiplicado por cinco para acoger a los pacientes enfermos. Según datos publicados por el Ministerio de Sanidad de España, a fecha de 27 de abril de 2020 había un total de 210.773 casos confirmados por técnica PCR, 23.822 fallecidos y 102.548 curados $^{1,2}$.

Los casos graves de COVID-19 presentan una insuficiencia respiratoria aguda que puede agravarse y precisar ventilación mecánica. Muchos de estos pacientes precisarán la realización de traqueotomías, que están catalogadas como procedimientos que generan aerosoles, por lo que conllevan un alto riesgo de contagio ${ }^{3,4}$.

\section{TRAQUEOTOMÍAS EN PACIENTES COVID-19}

\section{Justificación de traqueotomía en COVID-19}

La reciente pandemia por coronavirus COVID-19 está incrementando el número de pacientes que, debido a su situación ventilatoria pulmonar, pueden requerir de intubación orotraqueal muy prolongada. En estos pacientes, a medio plazo, el manejo de la vía aérea mejora con la realización de traquetomías. La infección por coronavirus COVID-19 ha demostrado una alta tasa de transmisibilidad, sobre todo por vía respiratoria y por dispersión de microgotas. Es por ello que la realización de una traqueotomía en pacientes COVID-19 es un procedimiento de alto riesgo de infección ${ }^{5-7}$.

En la UVI de nuestro hospital los pacientes en los que la intubación se prolonga durante más de 10-12 días son candidatos para realización de traqueotomía por el elevado riesgo de lesión de cuerdas vocales y laringe por la intubación prolongada.

Habitualmente, los médicos especialistas en cuidados intensivos realizan las traqueotomías percutáneas en aquellos pacientes en los que está indicado tal procedimiento (pacientes no obesos, cuellos "no complicados", pacientes más estables dentro de su gravedad, etc.). Durante el brote epidémico, el Hospital Universitario La Paz de Madrid ha sido durante semanas el epicentro de la infección por coronavirus 
en España, lo que se ha traducido en una saturación y bloqueo completo de las unidades de cuidados intensivos y quirófanos, que han sido reconvertidos en unidades de críticos. En esta situación se ha multiplicado el número de pacientes con anatomía cervical compleja y situación respiratoria extrema que ha requerido la realización de traqueotomías abiertas por parte de los servicios de Cirugía Oral y Maxilofacial, ORL y Cirugía Torácica.

Cuando se planifica una traqueotomía en un paciente COVID-19 intubado son muchas las consideraciones que debemos tener en cuenta.

En primer lugar, debemos considerar los parámetros de seguridad y riesgo de transmisión para el personal sanitario, tanto por contacto como por la inhalación de aerosoles. En este sentido nos enfrentamos a una situación jamás vivida por nuestro sistema sanitario que presenta todavía muchas cuestiones por resolver que no han sido claramente protocolizadas.

En segundo lugar, no tenemos una clara evidencia del beneficio de la realización precoz de una traqueotomía en pacientes COVID-19 debido a los pocos datos disponibles hasta la fecha. La estenosis laringo-traqueal posterior a la intubación es una complicación bien conocida en intubaciones prolongadas, pero no está demostrado que se reduzca significativamente en traqueotomías tempranas en revisiones sistemáticas (generalmente menos de 10 días) ${ }^{8}$. Por otro lado, en situaciones normales, la duración de la ventilación mecánica en pacientes críticos se reduce en casos de traqueotomía precoz y suele traducirse en estancias más cortas en unidades de cuidados intensivos, pero este dato no está todavía aclarado en estos pacientes. En cambio, el porcentaje de mortalidad global no suele verse modificado por este factor ${ }^{9}$.

No se ha identificado un momento concreto dentro de la evolución de los pacientes COVID-19 intubados en el que mejoran, permanecen estables o progresan hacia la muerte debido a las complicaciones pulmonares, y tampoco conocemos el impacto que tiene la traqueotomía en reclutar a los pacientes hacia esa eventual mejoría. Si nos fijamos en la epidemia de SARS-1, el tiempo medio desde el inicio de los síntomas hasta el fallecimiento de los pacientes fue de 23,7 días, lo que sugiere un pobre beneficio la realización de la traqueotomía antes de ese periodo de tiempo ${ }^{10}$. Los pacientes que no presentaron mejoría clínica ni remisión radiológica dentro de los 10 primeros días fueron los principales candidatos a precisar ventilación mecánica y a presentar una evolución más severa, llegando incluso hasta la muerte. No se estableció tampoco que la traqueotomía influyese en llevar a algunos pacientes hacia la mejoría de la función respiratoria, de manera que los criterios de traqueotomía fueron los mismos que para otros casos de intubación prolongada ${ }^{11}$.

En general, y aunque ha habido muchos ensayos controlados aleatorios sobre cuándo realizar la traqueotomía, la mayoría no posee una potencia estadística suficiente para detectar diferencias importantes, y las revisiones sistemáticas y metanálisis están limitados por la heterogeneidad de los estudios primarios ${ }^{12-15}$.

Finalmente, en esta situación de incertidumbre, hay otros aspectos importantes que debemos considerar, que son:

- Espacio físico donde realizar la traqueotomía.

- Recomendaciones generales.

- Medidas de protección para personal sanitario.
- Traslado de los pacientes a quirófano COVID-19.

- Equipo médico que realiza la traqueotomía.

- Tipo de traqueotomía a realizar.

- Tipo de cánula a emplear.

- Consideraciones especiales en traqueotomía COVID-19.

\section{¿Dónde realizar la traqueotomía?}

A pesar de que la traqueotomía es una técnica quirúrgica reglada, las características especiales de los pacientes COVID-19 hacen de este procedimiento una situación crítica por la inestabilidad pulmonar y la rápida desaturación del paciente, con dificultades de reclutamiento alveolar. En principio se considera que los traslados deben reducirse al mínimo imprescindible, aunque no existe un acuerdo respecto a este punto en los distintos protocolos publicados por múltiples sociedades médicas y organismos oficiales ${ }^{16-19}$.

Lo ideal sería su realización en la propia habitación aislada del paciente en la Unidad de cuidados críticos, a ser posible provista de un sistema de presión negativa. Esto evitaría el traslado del paciente al quirófano, con los riesgos de transmisibilidad para el personal y descompensación para el paciente. De hecho, esta es la gran ventaja de los procedimientos percutáneos in situ ${ }^{20,21}$. Cuando estas técnicas no son posibles y hay que recurrir a la traqueotomía abierta, nos encontramos con los siguientes problemas:

- La realización en la cama de UVI dificulta el acceso al paciente por la anchura de la cama.

- Se precisa una buena fuente de iluminación para realizar el procedimiento. En este sentido es posible el empleo de lámparas quirúrgicas portátiles con ruedas.

- Mayor dificultad para colocar al paciente en posición de hiperextensión.

- Falta de espacio para colocación de una mesa de mayo auxiliar.

En la situación actual, muchos quirófanos se han reconvertido en unidades de críticos. Esto facilita las posibilidades de realizar traqueotomías in situ, al contar con buena iluminación y estar cerca de los almacenes en los que se deposita el instrumental y material necesario para estos procedimientos. Por todo ello se deberá valorar en cada centro hospitalario el sitio más adecuado para llevar a cabo la cirugía en función de disponibilidad de quirófano cercano al área de críticos, personal para traslado y experiencia del equipo médico.

En el Hospital Universitario La Paz se han habilitado también dos quirófanos cercanos a las áreas de críticos, exclusivamente dedicados a las intervenciones de pacientes COVID-19, lo que ha permitido traslados cómodos de aquellos pacientes en los que se aconsejaba la intervención en un entorno quirúrgico. En estos quirófanos se ha establecido un circuito libre de COVID, donde los profesionales se visten para realizar el procedimiento y realizan un lavado quirúrgico, y otro COVID +, por donde los pacientes entran al quirófano. El problema es que la mayor parte de los quirófanos tienen presión positiva, lo que obliga a dejar abierta la comunicación con el antequirófano donde queda la cama del paciente. El resto de puertas, así como las "guillotinas" que comunican con las áreas de lavado y preparación de instrumental, deben quedar cerradas. 


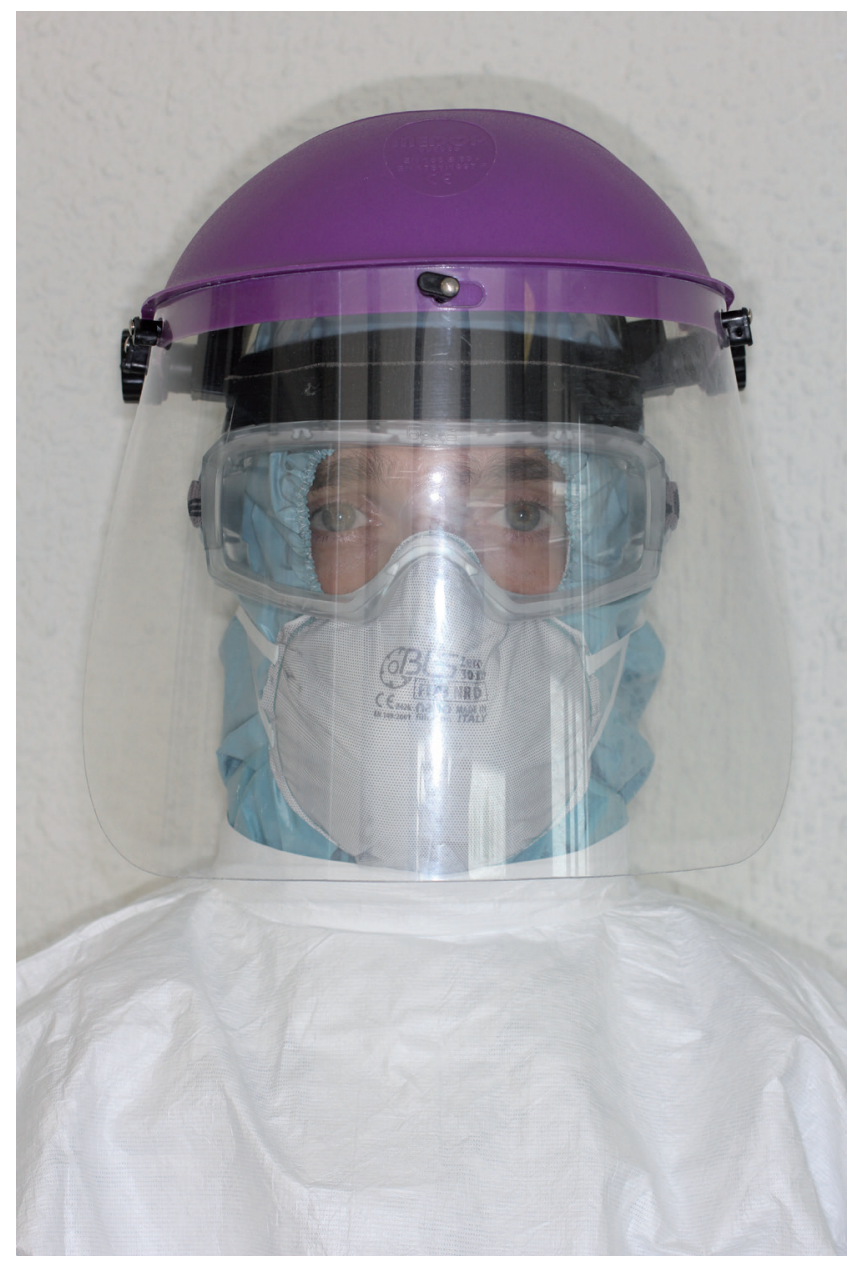

Figura 1. Equipo de protección personal con pantalla antisalpicaduras.

\section{Traslado de los pacientes a quirófano COVID-19}

El traslado de los pacientes desde la UVI hasta el quirófano debe programarse y contar con la coordinación entre intensivistas, equipo quirúrgico y personal de quirófano. No debemos olvidar que son pacientes que precisan una preparación especial para su traslado y movilización, como ya se ha comentado previamente $\mathrm{e}^{22,23}$.

Es por ese motivo por el que se deben crear grupos fijos de personal para dicha labor (intensivistas, anestesistas, enfermería y celadores) para realizar el traslado en el menor tiempo posible y estar preparados para cualquier eventualidad que en ocasiones obliga a detener el traslado (incluso en las zonas de tránsito entre la zona de críticos y el quirófano) ${ }^{24-27}$. Todo el personal responsable debe estar adecuadamente protegido con el equipo correspondiente y preparado para una situación de emergencia médica ${ }^{28}$.

\section{Medidas de protección para personal sanitario}

En cuanto a las medidas de protección, como en lo referente a la colocación y retirada de las mismas, sugerimos al

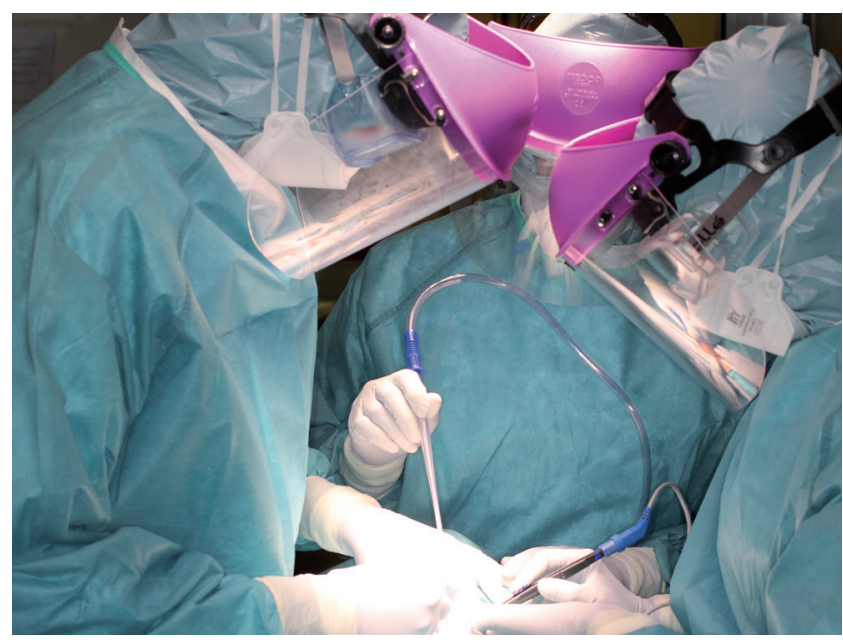

Figura 2. Imagen intraoperatoria de traqueotomía en paciente COVID-19 con tres cirujanos con los equipos de protección.

lector que consulte las recomendaciones de la SECOM-CyC, disponibles en su página web (www.secom.org).

En líneas generales, se aconseja prepararse adecuadamente con un EPI (equipo de protección individual) adecuado para evitar el contagio por virus respiratorios que conste de mascarillas de alta eficiencia de filtrado (FFP2-3), gafas integrales y pantalla antisalpicadura, así como bata impermeable (Figura 1).

\section{Equipo médico y preparación del quirófano}

A pesar de ser un procedimiento sencillo, se recomienda que sea realizado por facultativos con experiencia para disminuir el tiempo quirúrgico y poder enfrentarse a cualquier eventualidad. En principio, el número de personas en el quirófano debe ser el menor posible ${ }^{29-31}$. Minimizar el tiempo de exposición es clave para evitar el contagio durante el procedimiento ${ }^{32}$.

En caso de realizar la traqueotomía en la cama, se debe colocar en antitrendelemburg ligero, retirar almohada y colocarla debajo de los hombros. En el quirófano se hiperextenderá el cuello colocando un rodillo bajo los hombros, se retirarán los tubos y SNG hacia arriba manteniendo el campo libre y facilitando el acceso al tubo naso-orotraqueal para su retirada.

En el caso de realizar la intervención en el quirófano tenemos dos opciones, siempre con al menos dos cirujanos, anestesista y enfermera circulante (que proporcionará lo necesario tanto para el equipo quirúrgico como al anestesista durante la intervención):

- Opción 1: dos cirujanos y una enfermera instrumentista.

- Opción 2: tres cirujanos sin enfermera instrumentista (Figura 2).

La colocación es la habitual, con un cirujano a cada lado y el intensivista o anestesista en la cabecera. Para evitar la 


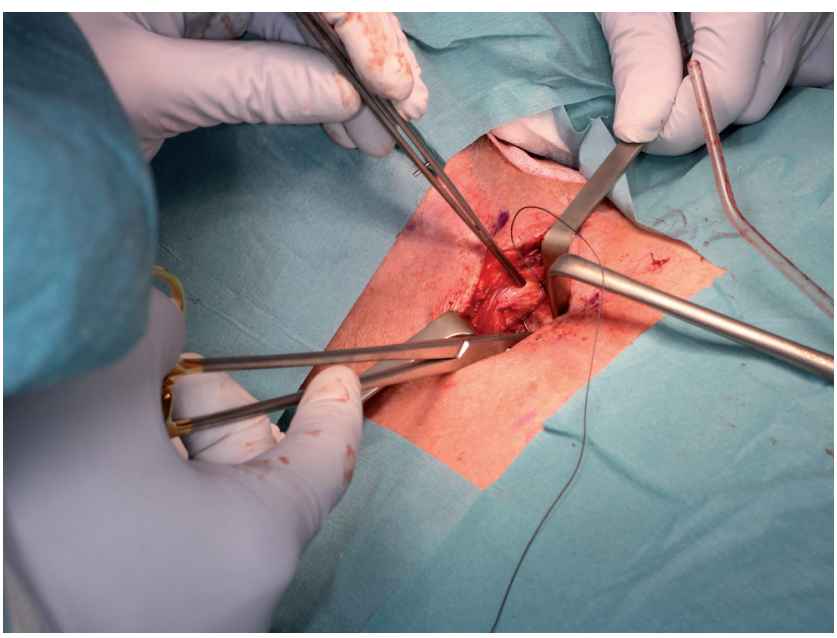

Figura 3. Punto de tracción al cartílago cricoides para facilitar la exposición de los anillos traqueales.

entrada de más personal que el estrictamente necesario, la mesa se colocará a la izquierda del paciente, siendo el ayudante el que instrumente. En caso de pacientes con cuello corto o mucho panículo adiposo cervical, puede ser recomendable un tercer cirujano que asista en las tareas de separación de tejidos y aspiración. Una maniobra que nos puede ayudar es la de colocar una cinta de esparadrapo anclada a la parte superior de la mesa, desde un lado de la cabeza al otro traccionando del mentón hacia atrás. Una vez que el paciente está colocado en la mesa de quirófano en decúbito supino, el equipo quirúrgico con el EPI colocado y revisado realizará un lavado de manos convencional y entrará al quirófano desde una "zona limpia" para, a continuación, colocarse la bata quirúrgica y los guantes estériles.

En todo momento la cavidad oral del paciente estará protegida o con una mascarilla o con un apósito adhesivo de vías para evitar la salida de saliva y/o aire que pueda fugar por la boca desde el sistema del respirador, lo que podría contagiarnos de forma directa.

En la medida de lo posible, y tal como aconsejan todas las guías en el manejo de la vía aérea de los pacientes COVID-1918, se deberán evitar sistemas de corte y coagulación eléctricos, ultrasónicos o de cualquier otro tipo que pueda esparcir macropartículas áreas y vaporización. Es preferible emplear bisturí convencional y sistemas de hemostasia convencionales, aunque en nuestro caso sí dispusimos de unas pinzas de bisturí eléctrico de coagulación bipolar, ya que el ayudante realizaba una aspiración de forma continua durante su uso.

\section{Técnica quirúrgica}

Nuestro protocolo incluye:

1. Incisión vertical en línea media para evitar el posible sangrado de las venas yugulares anteriores. Esta incisión nos permite una mejor exposición de la tráquea y menos dificultad para seccionar y ligar el tejido tiroideo.

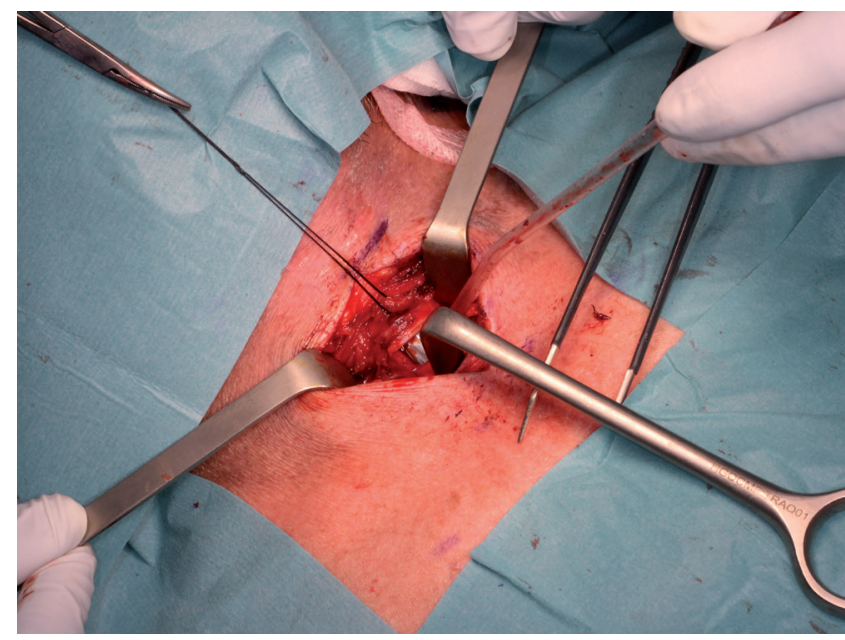

Figura 4. Ventana traqueal que permite visualizar el extremo del tubo orotraqueal, intentando no pinchar su balón.

2. Preferencia por la disección roma con mosquito y torunda. Los vasos más grandes se ligarán y los pequeños pueden cauterizarse con la pinza bipolar.

3. Ligadura convencional del itsmo tiroideo.

4. Disección traqueal desde el cricoides hasta el yugulum.

5. En caso de tráqueas de disposición vertical o con mucho panículo adiposo cervical, procedemos a aplicar un punto de lado a lado del cartílago cricoides que nos permitirá traccionar cranealmente del mismo, facilitando la exposición de los anillos traqueales, para proseguir con la técnica habitual (Figura 3).

6. Previamente a la incisión en la tráquea, y tras comprobar el balón de la cánula, el anestesista preoxigena al paciente hasta conseguir una saturación próxima al 100 \%. En ese momento, antes de realizar la apertura de la tráquea, el anestesista detiene la ventilación mecánica, a fin de evitar la salida masiva de aerosoles.

7. El tipo de incisión depende de la experiencia y preferencias de cada cirujano. En nuestro caso realizamos una ventana convencional entre $2 .^{\circ}$ o $3 .^{\text {er }}$ anillos traqueales, intentando en todo momento no perforar el balón del tubo orotraqueal (Figura 4). La retirada secuencial del tubo y la colocación de la cánula deben ir seguidas de la rápida reanudación de la ventilación mecánica.

8. Este procedimiento de canulación debe realizarse lo más rápido posible. Si se complica, es conveniente colocar un tubo flexo-metálico de forma temporal hasta que el anestesista consiga una buena saturación de $\mathrm{O}_{2}$. Tras esa estabilización se procede a la colocación rápida de la cánula definitiva (Figura 5).

9. Tras comprobación de la correcta ventilación del paciente conviene suturar parcialmente la herida en monoplano y finalmente procedemos a la fijación de la cánula mediante varios puntos de sutura a la piel. Intentamos evitar un cierre hermético de la herida, lo que podría facilitar un enfisema subcutáneo en caso 


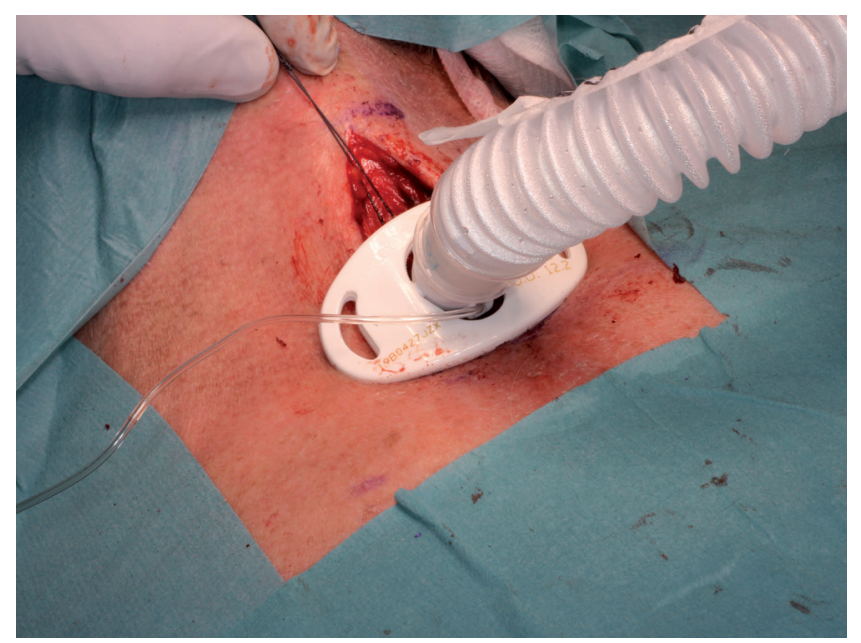

Figura 5. Cánula colocada y conectada al respirador antes de la sutura de la herida cutánea.

de fuga del balón de la cánula. Este acontecimiento se produce con relativa frecuencia en estos pacientes, debido a las altas presiones de ventilación mecánica.

En caso de realizar la traqueotomía en la UVI, para evitar la apertura traumática aconsejamos emplear el kit de traqueotomía percutánea. Para ello, un cirujano expondrá la tráquea y el otro pasará a la cabecera del paciente liberando la fijación del tubo oro-naso traqueal. El intensivista realizará las dilataciones sucesivas hasta colocar la cánula. El cirujano de la cabecera retirará lo suficiente el tubo para facilitar estas maniobras $^{33}$.

\section{Tipo de cánula a emplear. Consideraciones especiales en traqueotomía COVID-19}

Un aspecto importante a tener en cuenta es el tipo de cánula a emplear y su manera de inserción en la tráquea.

Es fundamental conocer las características técnicas de las cánulas (diámetro, longitud, curvatura, material, etc.) para realizar un procedimiento sin complicaciones ${ }^{34,35}$. El tamaño de la cánula corresponde a su diámetro interno (lo mismo sucede con los tubos orotraqueales). Debemos conocer sus diámetros, ya que las cánulas de diferente marca con un mismo diámetro interno pueden tener diferente diámetro externo.

Se debe elegir la curvatura de la cánula que más se adapte a la tráquea y el cuello del paciente, con el fin de evitar lesiones en la pared traqueal (Figura 6). Si la curvatura es muy corta, la cánula puede generar lesiones u obstruirse contra la pared posterior de la tráquea ${ }^{36-38}$. Las características de cada cánula suelen estar grabadas en las aletas de sujeción con el fin de poder ser visibles en todo momento.

Hay cánulas en las que podemos regular la longitud de la parte intra-extratorácica, que puede adaptarse mejor en pacientes con cuellos anchos y obesos. Este tipo de cánulas presentan, junto a las aletas de sujeción, una rosca que permite ajustar la altura para evitar su obstrucción, evitando el contacto directo con las paredes traqueales. Esta capacidad

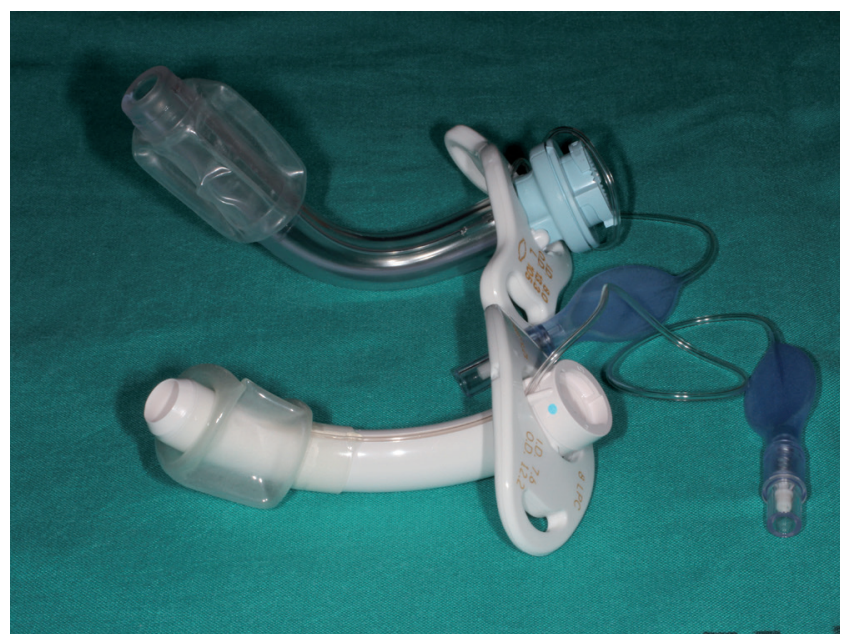

Figura 6. Curvaturas de las diferentes cánulas empleadas.

para su regulación puede ayudar específicamente a los intensivistas con los pacientes COVID-19 a la hora de colocarles en pronación.

En cuanto al material, las cánulas de plástico son muy utilizadas y pueden ser de polivinilo (PVC) o silicona. Las cánulas de PVC permiten que el tubo sea flexible, sin perder su forma, pero aumentan la posibilidad de adherencia de las secreciones y coágulos. Por el contrario, las de silicona, al ser menos porosas permiten menor adherencia a la pared interna del tubo. También existen unas cánulas flexometálicas, con una espiral de acero inoxidable en el medio de un cuerpo de silicona, lo que las otorga mayor flexibilidad y resistencia. Los anillos metálicos reducen la compresión y/o torsión de la cánula. Existen diferentes longitudes para adaptarse a cada caso en concreto.

\section{NUESTRA EXPERIENCIA}

Realizamos un estudio retrospectivo descriptivo de las traqueotomías realizadas por el servicio de Cirugía Oral y Maxilofacial del Hospital Universitario La Paz de Madrid, entre el 17 de marzo de 2020 y el 17 de abril de 2020 en pacientes COVID-19 de unidades de cuidados intensivos de nuestro hospital.

En total fueron 22 pacientes, siendo el rango de edad entre 40 y 77 años, con una media de edad de 64,9 años. De estos 22 pacientes, 18 (81,8 \%) fueron hombres y 4 (18,2 \%) fueron mujeres.

En cuanto a la sintomatología inicial en el momento del ingreso, los síntomas más comunes fueron:

- Fiebre en los 22 casos (100\%).

- Tos en 17 pacientes $(77,27 \%)$.

- Disnea en 15 casos (68,18\%).

Otros síntomas menos frecuentes fueron las mialgias $(36,36 \%)$, cefaleas (22,72 \%), molestias digestivas $(18,18 \%)$, diarrea $(13,63 \%)$, erupciones cutáneas $(9 \%)$ y dolor de garganta (9\%). 
Los principales antecedentes clínicos de los pacientes fueron:

- Obesidad en 17 pacientes (77,27\%).

- HTA en 14 casos (63,63\%).

- Dislipemia en 11 casos (50\%)

- Diabetes en 7 (31,81\%)

- EPOC en 4 casos (18,18\%).

- Antecedentes oncológicos en tres casos (13,63\%).

Hay que destacar que el $77 \%$ de los pacientes presentaban obesidad y la mitad dislipemia.

El tiempo transcurrido desde la fecha de inicio de síntomas hasta la intubación de los pacientes osciló entre 3 y 23 días, con una media de 10,72 días. El tiempo transcurrido desde la intubación hasta la realización de la traqueotomía de los pacientes osciló entre 8 y 21 días, con una media de 14,27 días.

En dos pacientes la traqueotomía se realizó en la UVI, en la propia cama del paciente, por grave inestabilidad de los mismos que desaconsejaban su traslado a quirófano.

En 21 de los 22 casos se realizó una traqueotomía a nivel del segundo anillo traqueal y tan solo en un caso en el tercer anillo traqueal. El tiempo quirúrgico medio fue de $9 \mathrm{minu}-$ tos y 40 segundos, si bien los tiempos de traslado, colocación en quirófano, fueron más tediosos de lo habitual. En todos los casos se realizó una incisión vertical para evitar el riesgo de sangrado de las venas yugulares anteriores y en todos los casos se empleó un punto de tracción al cartílago cricoides para facilitar la exposición de los anillos traqueales.

Respecto a las cánulas empleadas, utilizamos en 18 casos una cánula Shiley® (del número 6 en tres casos y en 12 casos cánulas del número 8). En los otros cuatro casos restantes empleamos una cánula flexometálica con regulación de la parte intra-extratorácica. La elección se realizó en función de la profundidad de la tráquea respecto a la piel y las dimensiones transversales de la misma.

En cuanto a las complicaciones intraoperatorias, en cuatro casos fue necesario emplear un tubo flexometálico previo a la cánula por desaturación brusca del paciente tras abrir la ventana traqueal, y sustituirla por la cánula definitiva tras remontar la saturación de oxígeno. En un caso el paciente sufrió un neumotórax masivo que precisó colocación inmediata de tubos de drenaje torácicos, pero finalmente el paciente falleció a los pocos minutos. En otro caso el paciente sufrió una desaturación brusca durante el traslado del quirófano a la UVI y falleció a los pocos minutos.

Respecto a las complicaciones tempranas, fue precisa la sustitución de una cánula aproximadamente a la hora de regreso a la UVI por fuga del balón de la cánula, realizándose el procedimiento en la propia cama de UVI del paciente. En otros dos casos los pacientes desarrollaron neumotórax con enfisema subcutáneo, posiblemente por salida de aire desde la tráquea. Se trataba de pacientes que habían requerido ventilación a muy altas presiones en los que el único factor de riesgo era una discreta traqueomalacia documentada durante el procedimiento quirúrgico.

Hasta la fecha de envío del artículo, solo un paciente, de 49 años, había recibido el alta de la unidad de cuidados intensivos, reforzando la conclusión de la necesidad de realizar la traqueotomía en estos pacientes por la necesidad de intubación prolongada. En este paciente se iniciaron cuidados regla- dos del traqueostoma en planta, tanto antes como después de su decanulación.

De igual forma, y exceptuando aquellos casos en los que se ha producido alguna alteración de la cánula, no fueron precisos recambios de las mismas. En este sentido es imprescindible un correcto manejo en las unidades de críticos, pues los cambios de cánula debe diferirse lo más posible para evitar desaturaciones bruscas en pacientes que se encuentran respiratoriamente muy comprometidos. Nuestro protocolo incluye siempre un punto de tracción del cricoides que puede ser útil en los cambios de cánula o en las desinserciones accidentales que pueden ocurrir al colocar a estos pacientes en posición de prono ${ }^{39}$.

El número total de exitus en nuestra serie ha sido de 6 pacientes (27,27\%), dos por complicaciones quirúrgicas/post inmediato y otros 4 pacientes durante su ingreso en la UVI. El tiempo transcurrido entre la traqueotomía y el fallecimiento osciló entre 7 y 14 días post-traqueo (con una media de 11 días).

Finalmente, es importante notificar que en el total de los procedimientos intervinieron cinco cirujanos y un anestesiólogo y hasta el día de remisión del artículo, ninguno había presentado síntomas de la enfermedad.

\section{CONCLUSIONES}

A pesar de los pocos meses que han transcurrido desde el inicio de la pandemia, contamos con muchas guías y documentos de sociedades científicas que expongan las medidas necesarias y los pasos a seguir para la realización de las traqueotomías en pacientes COVID-19. Aun así, muy pocas de ellas están basadas en la experiencia personal de los autores. En nuestra corta experiencia, podemos concluir lo siguiente:

- La traqueotomía será necesaria en casi todos los pacientes intubados, ya que se prevén periodos largos de ventilación mecánica.

- El procedimiento debe ser cuidadosamente planificado, estableciendo un circuito reglado que implique a todos los estamentos que van a participar para poder consensuar cada paso del proceso.

- Las maniobras técnicas deben ser lo más precisas posibles para evitar desaturaciones que dificulten el reclutamiento alveolar.

- Las medidas de protección son fundamentales para evitar el contagio como así lo hemos constatado en nuestra experiencia personal.

- En lo que se refiere a los pacientes, el sexo masculino, la obesidad, la HTA, la dislipemia y la diabetes, son los factores que parecen más asociados a fracaso respiratorio, intubación prolongada y necesidad de traqueostomía.

Estudios más amplios y con más tiempo de evolución permitirán sacar conclusiones a largo plazo más allá de esta experiencia preliminar en el momento más álgido de la pandemia.

\section{DECLARACIÓN DE CONFLICTO DE INTERESES}

Los autores declaran no tener conflictos de intereses y no tienen intereses en cualquier producto o dispositivo mencionado en este manuscrito. 


\section{AGRADECIMIENTOS}

Dedicado a todos los pacientes COVID-19 y sus cuidadores, que lucharon juntos durante el brote epidémico de marzoabril de 2020 y muy especialmente al Dr. Joaquín Díaz Domínguez, Jefe de Servicio de Cirugía General, que perdió la vida al servicio de su Hospital y sus pacientes.

\section{BIBLIOGRAFÍA}

1. Chan JF, Yuan S, Kok KH, To KK, Chu H, Yang J, et al. A familial cluster of pneumonia associated with the 2019 novel coronavirus indicating person-to-person transmission: a study of a family cluster. Lancet. 2020;395(10223):514-23. DOI: 10.1016/ S0140-6736(20)30154-9.

2. Ministerio de Sanidad del Gobierno de España. Actualización $\mathrm{n}^{\circ}$ 89. Enfermedad por el coronavirus (COVID-19); 28.04.2020. Disponible en: https:/www.mscbs.gob.es/profesionales/saludPublica/ccayes/alertasActual/nCov-China/documentos/Actualizacion_89_COVID-19.pdf

3. Peeri NC, Shrestha N, Rahman MS, Zaki R, Tan Z, Bibi S, et al. The SARS, MERS and novel coronavirus (COVID-19) epidemics, the newest and biggest global health threats: what lessons have we learned? Int J Epidemiol. 2020 Feb 22. pii: dyaa033. doi: 10.1093/ije/dyaa033. [Ahead of print].

4. Qiu H, Tong Z, Ma P, Hu M, Peng Z, Wu W, et al. China Critical Care Clinical Trials Group (CCCCTG). Intensive care during the coronavirus epidemic. Intensive Care Med. 2020;46(4):576-8. DOI: 10.1007/s00134-020-05966.

5. Morgan P. Tracheostomy in a patient with SARS. Br J Anaesth. 2004;92(6):905-6. DOI: 10.1093/bja/aeh568.

6. Ahmed N, Hare GM, Merkley J, Devlin R, Baker A. Open tracheostomy in a suspect severe acute respiratory syndrome (SARS) patient: brief technical communication. Can J Surg. 2005;48(1):68-71.

7. Kwan A, Fok WG, Law KI, Lam SH. Tracheostomy in a patient with severe acute respiratory syndrome. Br J Anaesth. 2004;92(2):280-2. DOI:10.1093/bja/aeh035.

8. Curry SD, Rowan PJ. Laryngotracheal Stenosis in Early vs Late Tracheostomy: A Systematic Review. Otolaryngol Head Neck Surg. 2020;162(2):160-7. DOI: 10.1177/0194599819889690.

9. Murthy S, Gomersall CD, Fowler RA. Care for Critically Ill Patients With COVID-19. JAMA. 2020;323(15):1499-500. DOI: 10.1001/jama.2020.3633.

10. Durbin CG. Early complications of tracheostomy. Respir Care. 2005;50(4):511-5.

11. Meininger D, Walcher F, Byhahn C. Tracheostomy in intensive care long-term ventilation: indications, techniques and complications. Chirurg. 2011;82(2):107-10, 112-5. DOI: 10.1007/ s00104-010-1972-9.

12. Boran OF, Bilal B, Bilal N, Öksüz H, Boran M, Yazar FM. Comparison of the efficacy of surgical tracheostomy and percutaneous dilatational tracheostomy with flexible lightwand and ultrasonography in geriatric intensive care patients. Geriatr Gerontol Int. 2020;20(3):201-5. DOI: 10.1111/ggi.13859.

13. Gobatto AL, Besen BA, Tierno PF, Mendes PV, Cadamuro F, Joelsons $\mathrm{D}$, et al. Comparison between ultrasound- and bronchoscopy-guided percutaneous dilational tracheostomy in critically ill patients: a retrospective cohorte study. J Crit Care. 2015;30(1):220.e13-7. DOI: 10.1016/j.jcrc.2014.09.011.

14. Iftikhar IH, Teng S, Schimmel M, Duran C, Sardi A, Islam S, et al. A Network Comparative Meta-analysis of Percutaneous Dilatational Tracheostomies Using Anatomic Landmarks, Bronchoscopic, and Ultrasound Guidance Versus Open Surgi- cal Tracheostomy. Lung. 2019;197(3):267-75. DOI: 10.1007/ s00408-019-00230-7.

15. Andriolo BN, Andriolo RB, Saconato H, Atallah ÁN, Valente O. Early versus late tracheostomy for critically ill patients. Cochrane Database Syst Rev. 2015;1(1):CD007271. DOI: 10.1002/14651858.CD007271.pub3.

16. Meng L, Qiu H, Wan L, Ai Y, Xue Z, Guo Q, et al. Intubation and Ventilation amid the COVID-19 Outbreak: Wuhan's Experience. Anesthesiology. 2020;10.1097/ALN.0000000000003296. DOI:10.1097/ALN.0000000000003296.

17. Broderick D, Kyzas P, Sanders K, Sawyerr A, Katre C, Vassiliou L. Surgical tracheostomies in Covid-19 patients: important considerations and the "5Ts" of safety. Br J Oral Maxillofac Surg. 2020. pii: S0266-4356(20)30158-3. DOI: 10.1016/j. bjoms.2020.04.008. [Ahead of print].

18. Takhar A, Walker A, Tricklebank S, Wyncoll D, Hart N, Jacob T, et al. Recommendation of a practical guideline for safe tracheostomy during the COVID-19 pandemic. Eur Arch Otorhinolaryngol. 2020. DOI: 10.1007/s00405-020-05993-x. [Ahead of print]

19. Tran K, Cimon K, Severn M, Pessoa-Silva CL, Conly J. Aerosol Generating Procedures and Risk of Transmission of Acute Respiratory Infections to Healthcare Workers: A Systematic Review. PLoS One. 2012;7(4):e35797. DOI: 10.1371/journal. pone.0035797.

20. Cox CE, Carson SS, Holmes GM, Howard ABS, Carey TS. Increase in tracheostomy for prolonged mechanical ventilation in North Carolina, 1993-2002. Crit Care Med. 2004;32(11):2219-26. DOI: 10.1097/01.CCM.0000145232.46143.40.

21. Brass P, Hellmich M, Ladra A, Ladra J, Wrzosek A. Percutaneous techniques versus surgical techniques for tracheostomy. Cochrane Database Syst Rev. 2016;7(7):CD008045. DOI: 10.1002/14651858.CD008045.pub2.

22. Chee VW, Khoo ML, Lee SF, Lai YC, Chin NM. Infection Control Measures for Operative Procedures in Severe Acute Respiratory Syndrome-related Patients. Anesthesiology. 2004;100(6):1394-8. DOI: 10.1097/00000542-200406000-00010.

23. Moore D, Gamage B, Bryce E, Copes R, Yassi A; BC Interdisciplinary Respiratory Protection Study Group. Protecting health care workers from SARS and other respiratory pathogens: organizational and individual factors that affect adherence to infection control guidelines. Am J Infect Control. 2005;33(2):8896. DOI: 10.1016/j.ajic.2004.11.003.

24. Smith JD, MacDougall CC, Johnstone J, Copes RA, Schwartz B, Garber GE. Effectiveness of N95 respirators versus surgical masks in protecting health care workers from acute respiratory infection: a systematic review and meta-analysis. CMAJ. 2016;188(8):567-74. DOI: 10.1503/cmaj.150835.

25. Zamora JE, Murdoch J, Simchison B, Day AG. Contamination: a comparison of 2 personal protective systems. CMAJ. 2006;175(3):249-54. DOI: 10.1503/cmai.060094.

26. Radonovich LJ Jr, Simberkoff MS, Bessesen MC, Brown AC, Cummings DA, Gaydos CA, et al. N95 Respirators vs Medical Masks for Preventing Influenza Among Health Care Personnel: A Randomized Clinical Trial. JAMA. 2019;322(9):824-33. DOI: 10.1001/jama.2019.11645.

27. Novak D. Why, where, and how PAPRs are being used in Health care. In: Institute of Medicine. The Use and Effectiveness of Powered Air Purifying Respirators in Health Care: Workshop Summary. 2015. Washington, DC: National Academies Press; 2015. Disponible en: https://www.ncbi.nlm.nih. gov/books/.

28. Government of Canada. Infection prevention and control for novel coronavirus (2019-nCoV): interim guidance for acute healthcare settings. Disponible en: https://www.canada.ca/en/ public-health/services/diseases/2019-novel-coronavirus-infection/health-professionals/infection-prevention-control-covid19-second-interim-guidance.html. 
29. Wei WI, Tuen HH, Ng RW, Lam LK. Safe tracheostomy for patients with severe acute respiratory syndrome. Laryngoscope. 2003;113(10):1777-9. DOI: 10.1097/00005537-200310000-00022.

30. Chee VW, Khoo ML, Lee SF, Lai YC, Chin NM. Infection control measures for operative procedures in severe acute respiratory syndrome-related patients. Anesthesiology. 2004;100(6):13948. DOI: $10.1097 / 00000542-200406000-00010$.

31. Wu Z, McGoogan JM. Characteristics of and Important Lessons From the Coronavirus Disease 2019 (COVID-19) Outbreak in China: Summary of a Report of 72314 Cases From the Chinese Center for Disease Control and Prevention. JAMA. 2020;323(13): 1239-1242. DOI:10.1001/jama.2020.2648.

32. D`Ascanio L, Latini G, Pandolfini M, Giardini D. Corona-steps for tracheotomy in COVID-19 patients: A staff-safe method for airway Management. Oral Oncol. 2020; 20:104731. DOI: 10.1016/j.oraloncology.2020.104731.

33. Ti, LK, Ang LS, Foong TW, Ng BS. What we do when a COVID- 19 patient needs an operation: operating room preparation and guidance. Can J Anesth. 2020;67:1-3. DOI: 10.1007/s12630-02001617-4.

34. Kwan A, FokWG, Law KI, Lam SH. Tracheostomy in a patient with severe acute respiratory syndrome. Br J Anaesth. 2004;92(2):280-2. DOI: 10.1093/bja/aeh035.

35. van Doremalen N, Bushmaker T, Morris DH, Holbrook MG, Gamble A, Williamson BN, et al. Aerosol and Surface Stability of SARS-CoV-2 as Compared with SARS-CoV-1. N Engl J Med. 2020;382(16):1564-7. DOI: 10.1056/NEJMc2004973.

36. Hess DR, N Altobelli NP. Tracheostomy Tubes. Respir Care. 2014;59(6):956-73; DOI: 10.4187/respcare.02920.

37. Lipton G, Steward M, McDermid R, Docking R, Urquhart C, MorrisonM, et al. Multispecialty tracheostomy experience. Ann $\mathrm{R}$ Coll Surg Engl. 2020;1:1-5. DOI: 10.1308/rcsann.2019.0184.

38. Meininger D, Walcher F, Byhahn C. Tracheostomy in intensive care long-term ventilation: indications, techniques and complications. Chirurg. 2011;82(2):107-10, 112-15. DOI: 10.1007/ s00104-010-1972-9.

39. González-Castro A, Escudero-Acha P, Peñasco Y, Leizaola O, Martínez de Pinillos Sánchez V, García de Lorenzo A. Intensive care during the 2019-coronavirus epidemic. Med Intensiva. 2020. pii: S0210-5691(20)30089-9. DOI: 10.1016/j.medin.2020.03.001. [Ahead of print].

\section{REFERENCIAS A PÁGINAS WEB}

1. Recomendaciones SECOM-CyC. http://www.secom.org/wpcontent/uploads/2020/03/1.-RECOMENDACIONES-GENERALESSECOMCYC-ACTUACION-COVID-19.pdf 